\title{
Controls for Solid-State Lighting
}

\author{
Final Report \\ Project M6643040 \\ Prepared for: \\ Joel Chaddock \\ National Energy Technology Laboratory \\ and \\ James Brodrick \\ Building Technologies Program \\ Office of Energy Efficiency and Renewable Energy \\ U.S. Department of Energy \\ Prepared by: \\ Francis Rubinstein \\ Lawrence Berkeley National Laboratory \\ 1 Cyclotron Rd., Building 90R3111 \\ Berkeley CA, 94720
}

June 2007 


\title{
Controls for Solid-State Lighting
}

\author{
Final Report \\ Prepared for: \\ Joel Chaddock \\ National Energy Technology Laboratory \\ and \\ James Brodrick \\ Building Technologies Program \\ Office of Energy Efficiency and Renewable Energy \\ U.S. Department of Energy
}

\author{
Prepared by: \\ Francis Rubinstein \\ Lawrence Berkeley National Laboratory \\ 1 Cyclotron Rd., Building 90R3111 \\ Berkeley CA, 94720
}

June 2007 


\section{DISCLAIMER}

This report was prepared as an account of work sponsored by an agency of the United States Government. Neither the United States Government, nor any agency thereof, nor any of their employees, nor any of their contractors, subcontractors, or their employees, makes any warranty, express or implied, or assumes any legal liability or responsibility for the accuracy, completeness, or usefulness of any information, apparatus, product, or process disclosed, or represents that its use would not infringe privately owned rights. Reference herein to any specific commercial product, process, or service by trade name, trademark, manufacturer, or otherwise, does not necessarily constitute or imply its endorsement, recommendation, or favoring by the United States Government or any agency, contractor or subcontractor thereof. The views and opinions of authors expressed herein do not necessarily state or reflect those of the United States Government or any agency thereof. 


\section{ACKNOWLEDGEMENTS}

The authors would like to the following individuals for their advice in the preparation of this report: Jim Galvin (LBNL), Dmitriy Bolotov (LBNL), Erik Page (California Lighting Technology Center), Terry Clark (Finelite), Andy Lippman (Next Generation Lighting), Paul Thurk, and James Brodrick (U.S. Department of Energy). This work was supported by the Assistant Secretary for Energy Efficiency and Renewable Energy, Building Technologies Program, of the U.S. Department of Energy under Contract No. DE-AC0205CH11231. 


\section{Table of Contents}

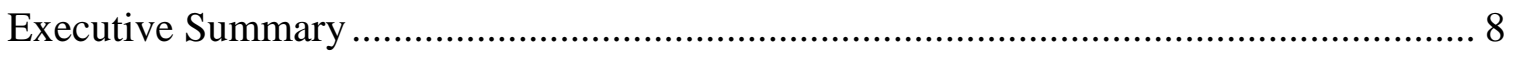

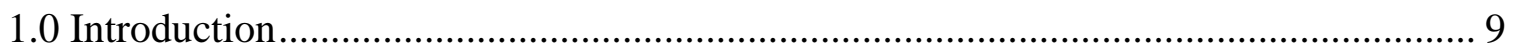

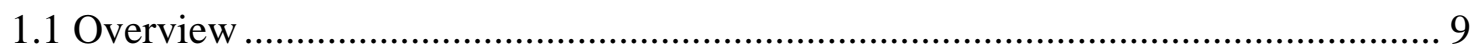

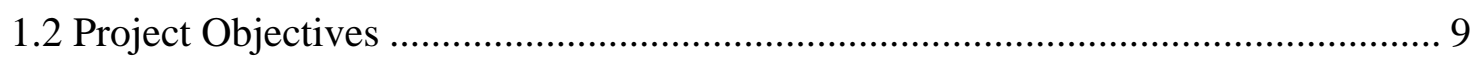

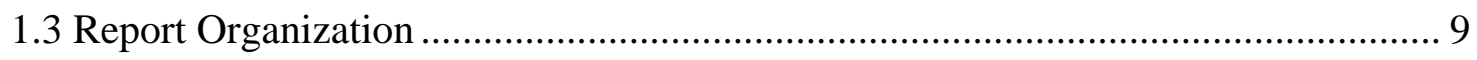

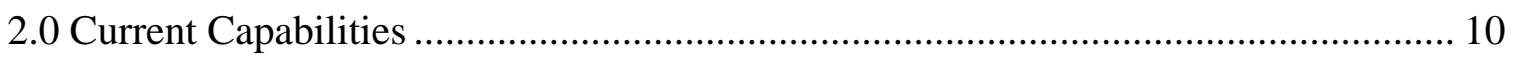

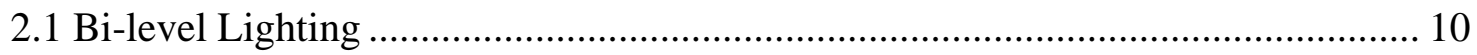

2.2 Commercially-Available LED Drivers …………............................................... 11

2.3 Comparing Dimming Efficiency of Constant Current and Pulse Width Modulation

12

2.4 Current State-of-the-Art in Dimming LED Luminaires............................................ 13

2.5. Integrated LED Tasklights .............................................................................. 13

2.6. New LED Hybrid Lighting Systems ...................................................................... 15

2.7. Efficiency of LED Drivers: Today and Projected.................................................. 15

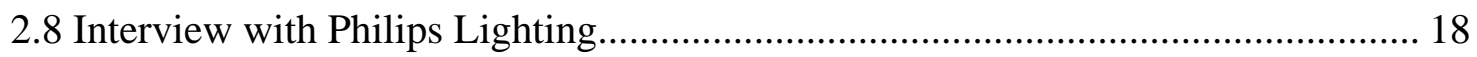

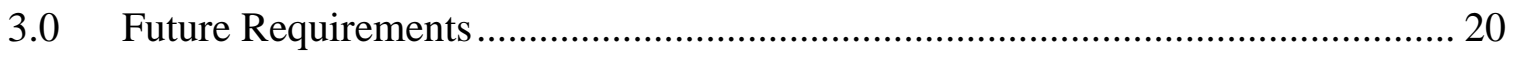

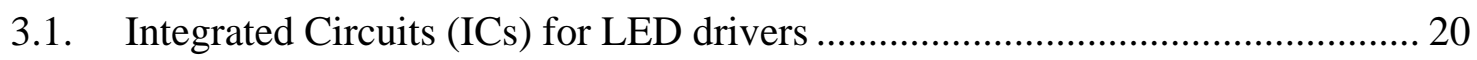

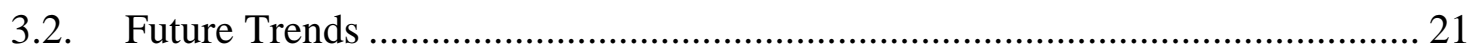

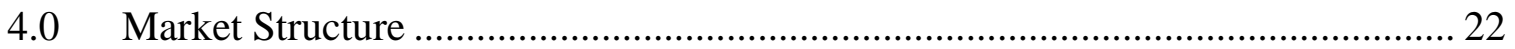

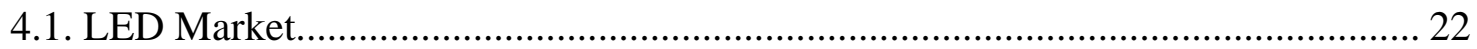

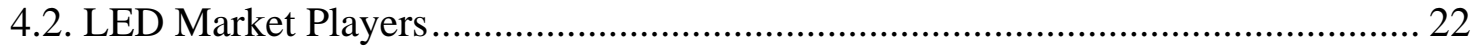

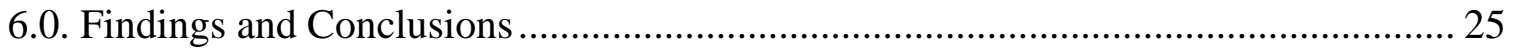

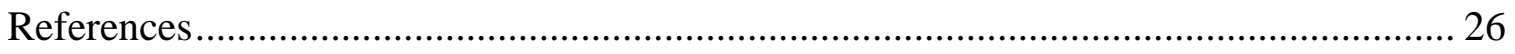

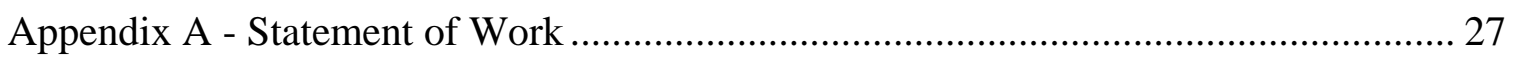

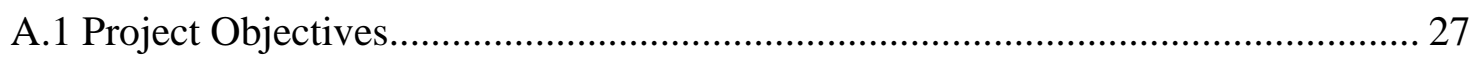




\section{List of Figures}

FiguRE 1. CiRCUIT DIAGRAM FOR CONSTANT CURRENT POWER SUPPLY

FIGURE 2. CIRCUIT DIAGRAM SHOWING DIMMING CONSTANT CURRENT DRIVER TAKING ITS INPUT FROM

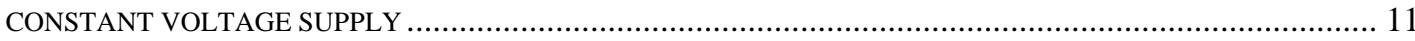

FIGURE 3. CONTROLLING THREE COLOR LED CHANNELS WITH CONSTANT VOLTAGE POWER SUPPLY ........... 12

FIGURE 4. EXAMPLE OF STATE-OF-THE-ART DIMMABLE LED LUMINAIRE CONTROLLED USING SIMPLE 0-10 VDC DIMMER.

FIGURE 5. CIRCUIT DIAGRAM SHOWING HOW LED LUMINAIRE IS DIMMED USING AVAILABLE OFF-THE-SHELF COMPONENTS. COURTESY ANDY LIPPMAN, NEXT GENERATION LIGHTING.

FIGURE 6. IMAGES OF THE LED TASK LIGHTING SYSTEM SHOWING 3 WATT LED TASKLIGHTS (A-C), INTEGRATED CONTROLLER (D), 9 WATT UNDERCOUNTER LED LIGHT (E) AND ENTIRE SYSTEM (F). COURTESY OF TERRY ClaRK, FinELITE CO.

FIGURE 8. PROJECTED IMPROVEMENT IN SYSTEM LUMINOUS EFFICACY OBTAINED BY RAISING DRIVER ELECTRICAL EFFICIENCY FROM CURRENT INDICATED LEVELS TO 94\% EFFICIENCY (MAXIMUM PRACTICALLY ACHIEVABLE LEVEL)

FiguRE 9. INTERNAL BOARD FOR ADVANCE LED DRIVER MODEL LED120A0350C33F

FIGURE 10. MANUFACTURER-LISTED EFFICIENCIES OF INTEGRATED CIRCUITS (ICS) INTENDED FOR PACKAGED LED DRIVERS (39 ICS FROM 5 MANUFACTURERS .. 20

FIGURE 11. THE CURRENT DISTRIBUTION OF LEDS BY MAJOR APPLICATION TYPE (LEFT PIE CHART) AND THE EXPECTED GROWTH IN EACH OF THE KEY MARKET SECTORS FOR THE NEXT 5 YEARS (RIGHT HAND PLOT).

FIGURE 12. LED MARKET CHAIN SHOWING MANUFACTURING STEPS FROM WAFER TO FINAL LIGHTING SYSTEM (FROM THURK 2006) 


\section{List of Tables}

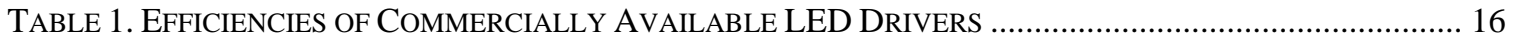

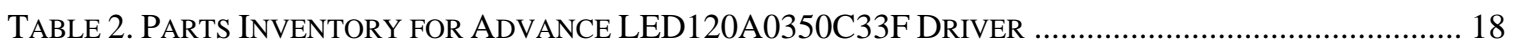

TABLE 3. LED MARKET ENTITIES AND MAIN MARKET Functions .............................................................. 23 


\section{Executive Summary}

This study predicts new hybrid lighting applications for LEDs. In hybrid lighting, LEDs provide a low-energy "standby" light level while another, more powerful, efficient light source provides light for occupied periods. Lighting controls will allow the two light sources to work together through an appropriate control strategy, typically motionsensing. There are no technical barriers preventing the use of low through high CRI LEDs for standby lighting in many interior and exterior applications today.

The total luminous efficacy of LED systems could be raised by increasing the electrical efficiency of LED drivers to the maximum practically achievable level (94\%). This would increase system luminous efficacy by $20-25 \%$. The expected market volumes for many types of LEDs should justify the evolution of new LED drivers that use highly efficient ICs and reduce parts count by means of ASICs. Reducing their electronics parts count by offloading discrete components onto integrated circuits (IC) will allow manufacturers to reduce the cost of LED driver electronics.

LED luminaire manufacturers will increasingly integrate the LED driver and thermal management directly in the LED fixture. LED luminaires of the future will likely have no need for separable lamp and ballast because the equipment life of all the LED luminaire components will all be about the same (50,000 hours).

The controls and communications techniques used for communicating with conventional light sources, such as dimmable fluorescent lighting, are appropriate for LED illumination for energy management purposes. DALI has been used to control LED systems in new applications and the emerging ZigBee protocol could be used for LEDs as well. Major lighting companies are already moving in this direction.

The most significant finding is that there is a significant opportunity to use LEDs today for standby lighting purposes. Conventional lighting systems can be made more efficient still by using LEDs to provide a low-energy standby state when lower light levels are acceptable. 


\subsection{Introduction}

\subsection{Overview}

The Department of Energy is supporting the development of solid state lighting technologies and systems through its Building Technologies Program (http://www.eere.energy.gov/buildings). Most of that effort is aimed at the fundamental research for improving the efficiency and reducing the cost of LED and OLED sources. Identifying new and emerging trends for electrically controlling these new sources is the purpose of this study.

\subsection{Project Objectives}

The objective of the project is to characterize and identify trends towards control, operation, and maintenance of Solid State Lighting systems, both today and as envisioned for tomorrow's lighting systems. The approach shall include a market reconnaissance study to identify the technical and market issues associated with the control, operation and maintenance of solid state lighting systems. The study scope is primarily limited to the control of LED sources but may include analysis of OLEDs as appropriate.

The Scope of Work for the Project is given in Appendix A.

\subsection{Report Organization}

The report has five sections. The first section describes the overall objective of the report, the objectives of each of the three major technical tasks and the report's structure. The next three sections of the report provide the results of each of the three tasks. The final section presents an analysis of the opportunities for using LEDs as "standby" lighting to add value to well-controlled conventional lighting systems. The final section presents the findings and conclusions of the study. 


\subsection{Current Capabilities}

The US Department of Energy is pursuing an aggressive technology development effort to develop new lighting systems built upon solid-state lighting. Solid state light sources consist of two broad classes - lighting emitting diodes (LEDs) and organic LEDs (OLEDs). [1] provides a comprehensive overview of the technical and economic hurdles faced by LED and OLED systems if the desired end result is to replace most conventional lighting for illumination purposes. [DOE 2006] divides the high brightness LED market into 4 quality classes: Low, Medium, High and Very High CRI ${ }^{1}$. Replacing general illumination will be a formidable challenge for LEDs because mature light sources, such as fluorescent lighting, already provide excellent illumination at a high CRI at a cost of pennies a footcandle. ${ }^{2}$

\subsection{Bi-level Lighting}

There have been developments in the lighting controls industry that may provide immediate cost-effective application of Medium and Low CRI LEDs for the purposes of providing "standby" lighting for many interior and exterior applications. The deliberate provision of a low-energy state for lighting systems is actually not new. California's Title 24 Non-Residential Building Code has required "bi-level” lighting for many categories of commercial spaces since 1982 [8]. As a result, much of California's commercial floor space has the capability of being switched to typically 50\% light level usually using two switches that are easily accessible to the users of the space. Use of bi-level switching in the State is estimated to have saved about 1 TWh annually Statewide, saving businesses and schools $\$ 100$ million a year.

Since 2003, several manufacturers have been producing luminaires for building stairwells that provide a low "standby" level during unoccupied times and then switch to full light output when occupancy is detected by the fixture's motion sensor. The standby level is often only $10 \%$ of the full level and as a result uses much less energy overall than would be the case if the stairwell were always lit at the high level as is conventional. Stairwells are often occupied only $5 \%$ of the time, so the provision of a low-energy standby state is a significant energy saving opportunity.

It is proposed that this is another unexplored opportunity to utilize LEDs not to replace fluorescent lighting but rather to add value to fluorescent lighting systems, especially in sparsely-occupied areas where a high light level is only required occasionally. There is no reason why LEDs cannot fulfill this role for at least some commercial applications such

\footnotetext{
${ }^{1}$ Low would eventually replace mercury and HPS, Medium CRI LEDs would replace cool white FL, high CRI LEDs might eventually replace RE phosphored lamps and CFLs, and very high would replace incandescent and halogen

${ }^{2}$ A modern specification grade fluorescent lighting system providing office light levels (500 lux or 50 footcandles) costs typically $\$ 3.50 /$ square foot. Thus the cost of providing illumination is $\$ 0.07 /$ footcandle.
} 
as stairwells, corridors and building exteriors. The requirements for LEDs at standby light levels are not challenging; they can be met by today's LEDs.

\subsection{Commercially-Available LED Drivers}

In order to characterize the performance of today's LED drivers, researchers performed a search for technical information on the performance of LED drivers from the two largest suppliers -- Osram Sylvania and Advance/Philips. These two companies are of particular interest because these companies produce not only the LED drivers but also the actual LEDs themselves.

These manufacturers have two basic types of LED driver: constant current and constant voltage power supplies. OSI's constant current power supplies are designed to work with their hi-flux LED modules (such as their “Dragon” LEDs, which are white, phosphored LEDs). For non-dimming applications, the constant current driver would pull power from directly from a 120 VAC line and have sufficient current capacity to drive $350 \mathrm{~mA}$ of LED load (see Figure 1).

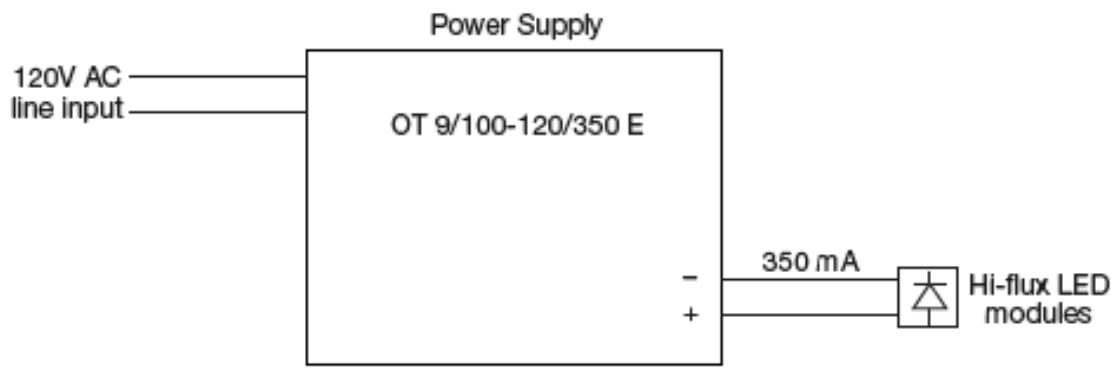

Figure 1. Circuit diagram for Constant Current power supply

For dimming applications, the situation is more complicated. In this case, the dimming constant current driver takes it input from a constant voltage power supply. The output of the connected $350 \mathrm{~mA}$ LED load is dimmed by the application of a 1-10 VDC signal to the dimming power supply (see Figure 2). The 1-10 VDC signal can be supplied by a DALI to 0-10 VDC converter, which opens the possibility of controlling LED lighting using the DALI protocol.

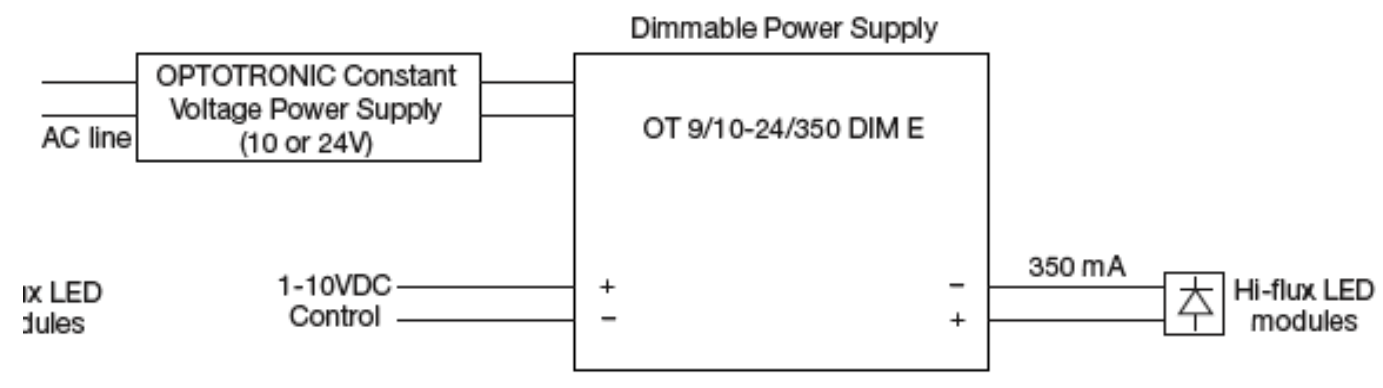

Figure 2. Circuit diagram showing dimming constant current driver taking its input from constant voltage supply 
Constant voltage power supplies are also being used especially for LED RGB applications where it is necessary to alter the amount of red, green and blue light for the application. For these applications, it is convenient to tie an electronic LED RGB control to a constant voltage power supply as shown in Figure 3:

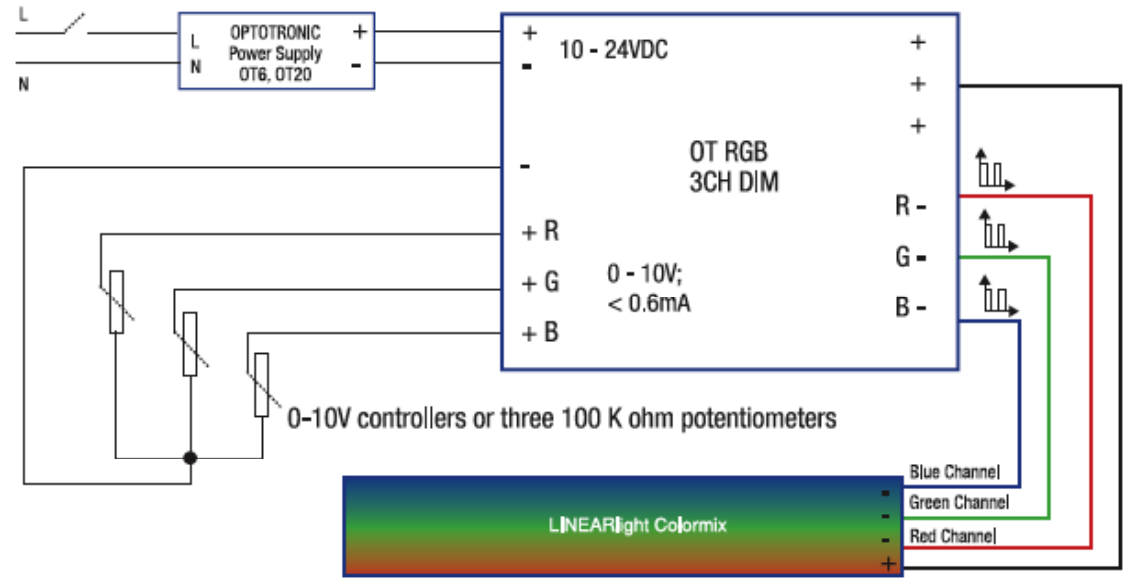

Figure 3. Controlling three color LED channels with constant voltage power supply

The dimming module uses pulse width modulation to "duty cycle" the red, green and blue channels thus altering the relative color output of the light.

\subsection{Comparing Dimming Efficiency of Constant Current and Pulse Width Modulation}

It is expected that there will be differences in the efficiencies associated with dimming white LED lighting depending on whether the LED source is dimmed using constant current reduction (CCR) or using pulse width modulation (PWM).

Gu et. al. [9] describes an experimental study conducted to quantify the spectral and luminous efficacy changes of high-powered and pc-white LEDs under different dimming methods. The paper finds that under constant current reduction (CCR) dimming, the efficacies of white LEDs increase with decreasing current (and junction temperature) reaching 150-200\% increase in efficacy (compared to efficacy at $350 \mathrm{~mA}$ ). As LED lamp current drops to under 10\% of the nominal $350 \mathrm{~mA}$ (35 mA), the efficacy decreases slightly. With pulse width modulation (PWM) dimming, the efficacies of white LEDs remained roughly constant (for two white LED manufacturers) or increased but less markedly (for a third manufacturer) until about $20-30 \%$ of nominal full duty cycle where efficacy drops for all manufacturers. Although the details are still murky it appears that the relative efficacy difference between CCR and PWM dimming can be higher than $100 \%$, with CCR dimming much more efficient than PWM dimming. This suggests that CCR will be the preferred efficient method for dimming white LEDs in the future. 


\subsection{Current State-of-the-Art in Dimming LED Luminaires}

As an example of current trends in LED control requirements, a state-of-the-art dimmable LED luminaire, which was presented at the 2007 Annual Conference of the Illuminating Engineering Society, is described. The Next Generation Lighting uses off-the-shelf dimming hardware to control the intensity of their "Bold Stripe" LED luminaire. As shown in Figure 4 below, the LED luminaire can be significantly dimmed using a simple $0-10$ VDC dimmer. The controls components required to dim the luminaire are shown in Figure 5.
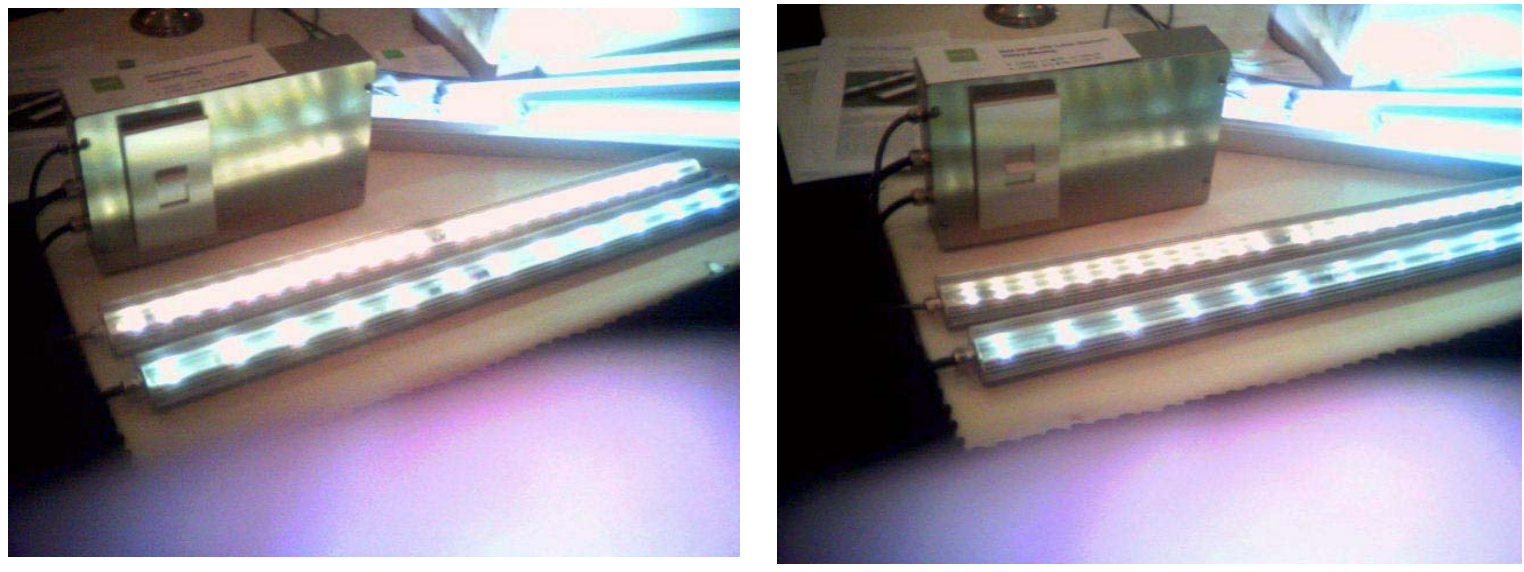

Figure 4. Example of state-of-the-art dimmable LED luminaire controlled using simple 0-10 VDC dimmer.

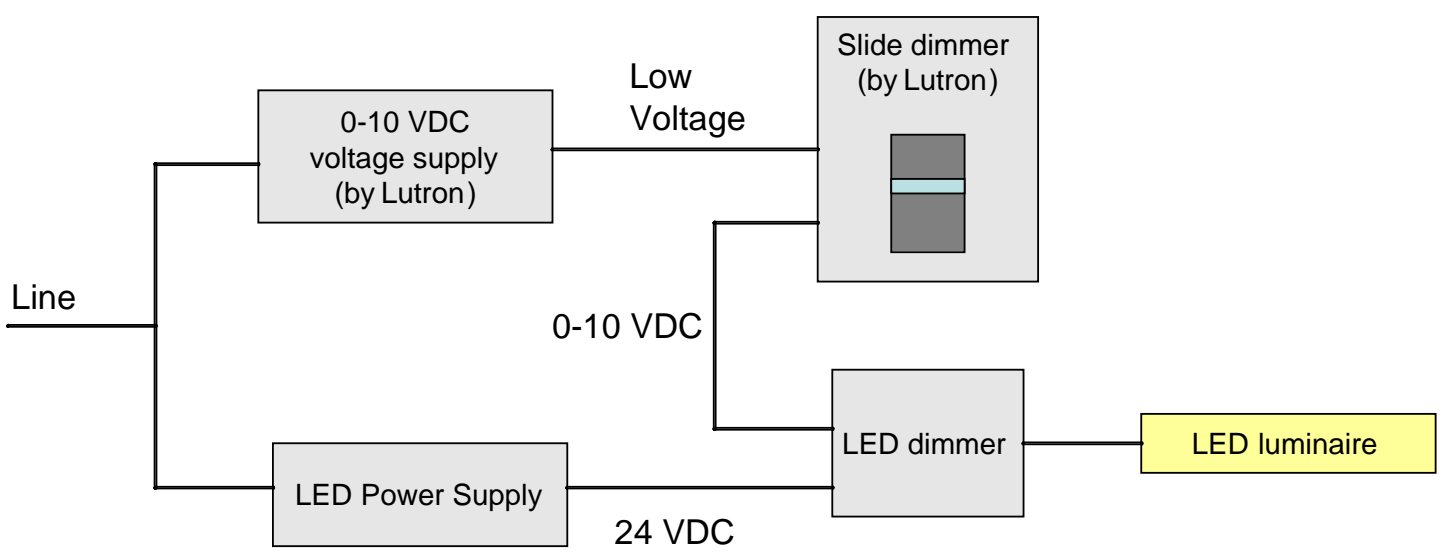

Figure 5. Circuit diagram showing how LED luminaire is dimmed using available off-the-shelf components. Courtesy Andy Lippman, Next Generation Lighting.

\subsection{Integrated LED Tasklights}

LEDs are starting to find application in high-end task lighting and desk lamps. As an example, Finelite has created a new LED workstation-based lighting system that is 
controlled from a small centralized power supply. The power supply and controller provides up to 20 watts of power for up to 3 LED task and under-counter lights. One controller (see Figure 6D) is required per workstation, and it can be controlled from a single workstation-based occupant sensor (see Figure 6F). This LED control and lighting system is representative of an emerging trend for office lighting, where local task lighting needs will be met by carefully-crafted LED lights that are controlled from a single, high efficiency power supply by an occupancy sensor (or photosensor).

A

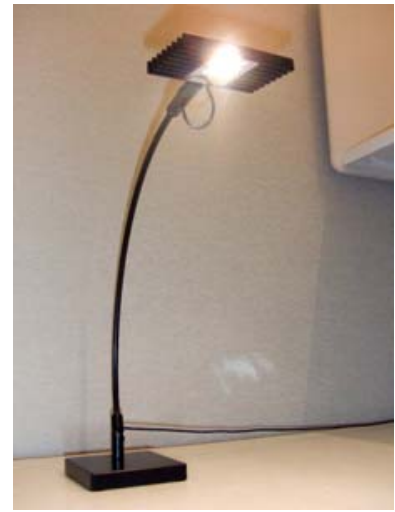

C

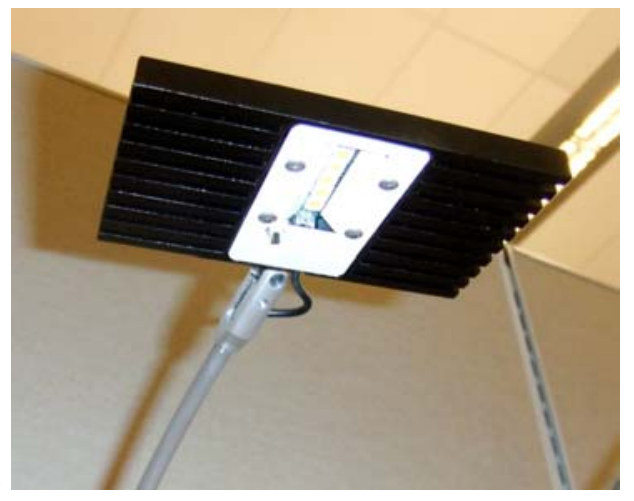

E

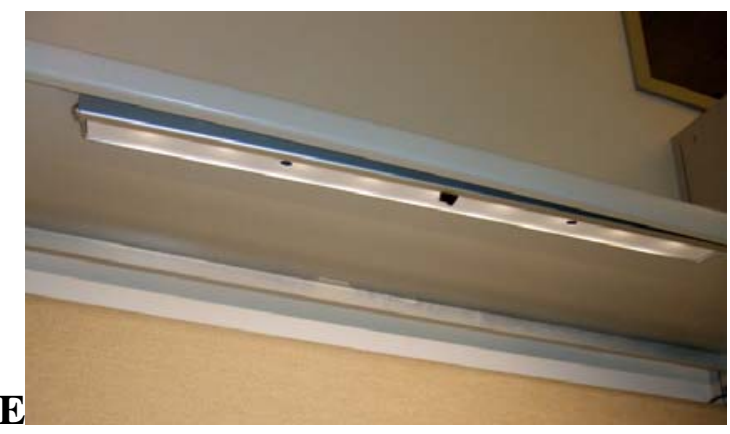

B

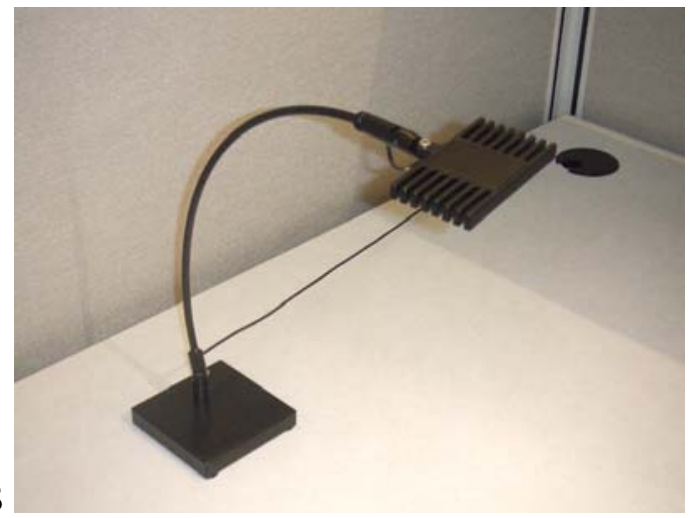

D
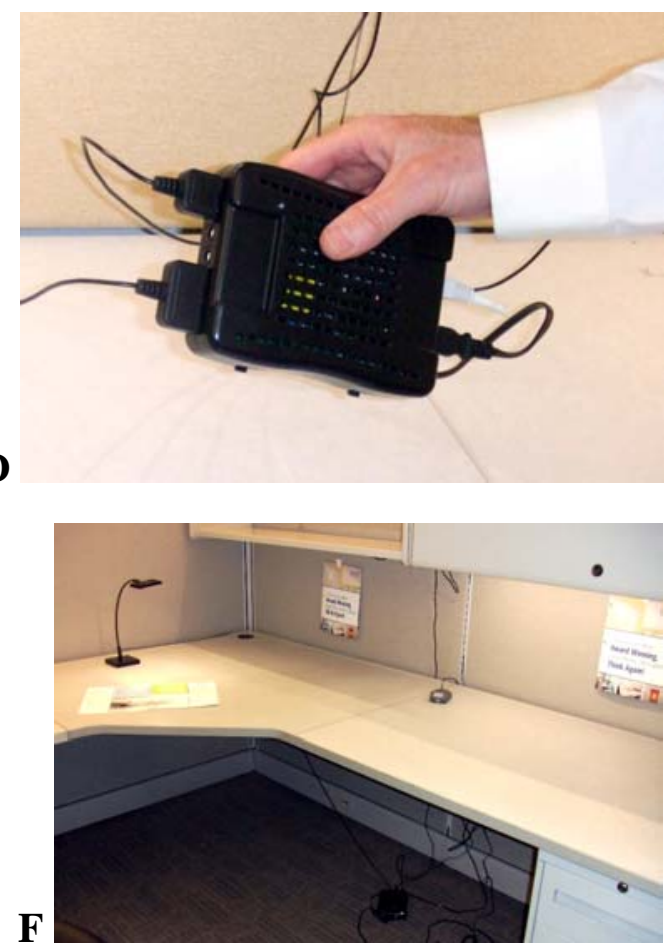

Figure 6. Images of the LED task lighting system showing 3 watt LED tasklights (AC), integrated controller (D), 9 watt undercounter LED light (E) and entire system (F). Courtesy of Terry Clark, Finelite Co. 


\subsection{New LED Hybrid Lighting Systems}

One particularly interesting development is the evolution of a new class of lighting systems that use a conventional light source for primary illumination and LEDs, for providing a low "standby" light level. Several luminaire manufacturers, with technical assistance of the California Lighting Technology Center at UC Davis, have begun to field these new systems.

Some examples of the promising hybrid LED lighting systems are:

\section{LED Hybrid Exterior Fixtures.}

This porch light uses an LED light to provide a low "standby" level during unoccupied periods. When motion is detected by the built-in motion sensor, the incandescent lamp switches on providing a high light level until the motion sensor times out.

\section{Hospitality/Bathroom Hybrid Lighting}

Another successful example of a hybrid LED lighting system, is a system fielded by the Wattstopper for the hospitality industry. In this patented application, conventional fluorescent bathroom lighting is placed under occupancy sensor control, which switches to low-level LED lighting after the occupancy sensor times out the conventional light.

\section{Outdoor Bollard Lighting}

Another company, Gardco, makes a 100\% LED outdoor bollard which switches between a high and low energy state when triggered by an integrated occupancy sensor. In this product, the LEDs provide both the high and low light level. This approach not only saves energy but also extends LED lamp life because when the bollard operates in low energy mode, there is far more heat sinking than is necessary to keep the junction temperature at reduced temperatures.

\subsection{Efficiency of LED Drivers: Today and Projected}

In this section, researchers characterize the electrical efficiency of LED drivers available today and project the potential improvements in overall system luminous efficacy that could be realized by raising the driver efficiencies to the maximum practically achievable level. In addition, researchers estimated the potential cost savings that could be realized by driver manufacturers if they reduce their electronics parts count by offloading discrete components onto integrated circuits (IC).

The scope of the investigation was restricted to drivers for white LEDs (as opposed to RGB LEDs) because these have the largest application for general illumination purposes. Both types of common drivers discussed previously - constant voltage and constant current - from the two largest driver manufacturers were examined.

The efficiencies of 13 different LED drivers were determined using listed data from the manufacturers' technical data sheets as shown in Table 1. 


\section{Table 1. Efficiencies of Commercially Available LED Drivers}

\begin{tabular}{|c|c|c|c|c|c|}
\hline Manufacturer & Type & Model Number & $\begin{array}{l}\text { Calculated } \\
\text { Efficiency }\end{array}$ & $\begin{array}{l}\text { System Efficacy } \\
\text { Improvement @ } \\
\text { 94\% efficiency }\end{array}$ & $\begin{array}{c}\text { Maximum System } \\
\text { Efficacy Improvement } \\
\text { (100\% efficiency) }\end{array}$ \\
\hline OSI & Voltage & OT 6/100-120/10 CE & $80.0 \%$ & 1.18 & 1.25 \\
\hline OSI & Voltage & OT 50/120-277/10E UNV1 & $87.0 \%$ & 1.08 & 1.15 \\
\hline OSI & Voltage & OT 6/100-120/24 CE & $80.0 \%$ & 1.18 & 1.25 \\
\hline OSI & Voltage & OT 20/120-240/24 S UNV1 & $87.0 \%$ & 1.08 & 1.15 \\
\hline OSI & Voltage & OT 75/120-277/24E UNV1 & $90.4 \%$ & 1.04 & 1.11 \\
\hline OSI & Current & OT 9/100-120/350 UNV1 & $73.9 \%$ & 1.27 & 1.35 \\
\hline Advance & Voltage & LED120A0012V10F & $80.0 \%$ & 1.18 & 1.25 \\
\hline Advance & Voltage & LED120A0012V50F & $80.0 \%$ & 1.18 & 1.25 \\
\hline Advance & Voltage & LED120A0024V07F & $79.1 \%$ & 1.19 & 1.26 \\
\hline Advance & Voltage & LED120A0024V10F & $79.9 \%$ & 1.18 & 1.25 \\
\hline Advance & Voltage & LED120A0024V33F & $80.0 \%$ & 1.18 & 1.25 \\
\hline Advance & Current & LED120A0350C33F & $80.0 \%$ & 1.18 & 1.25 \\
\hline Advance & Current & LED120A0700C24F & $80.0 \%$ & 1.18 & 1.25 \\
\hline
\end{tabular}

Of the 13 examined drivers, the lowest efficiency was 74\% (constant current type) while the highest efficiency was $90.4 \%$ (constant voltage type). Note that these estimates are based on the manufacturers listed data for input watts. It is likely that the efficiencies calculated in this manner are conservative and that the true efficiencies are actually higher by a couple of points. However, it is not possible to refine the estimates of driver efficiencies without performing physical measurements on real LED systems.

Manufacturers could improve the overall luminous efficacy of their white LED products by raising the electrical efficiency of the drivers. The maximum practically achievable driver efficiency is about $94 \%$, which will be difficult to achieve in practice. The main cause of driver loss is $\mathrm{I}^{2} \mathrm{R}$ losses in circuit resistances. Because LED drivers are inherently low voltage devices requiring at least one diode with forward voltage of 0.6 volts there are limits to how much driver losses can be decreased.

Figure 7 shows the projected improvements in system efficacy that would be achieved by raising all the above driver efficiencies from their current level to $94 \%$ efficiency. 


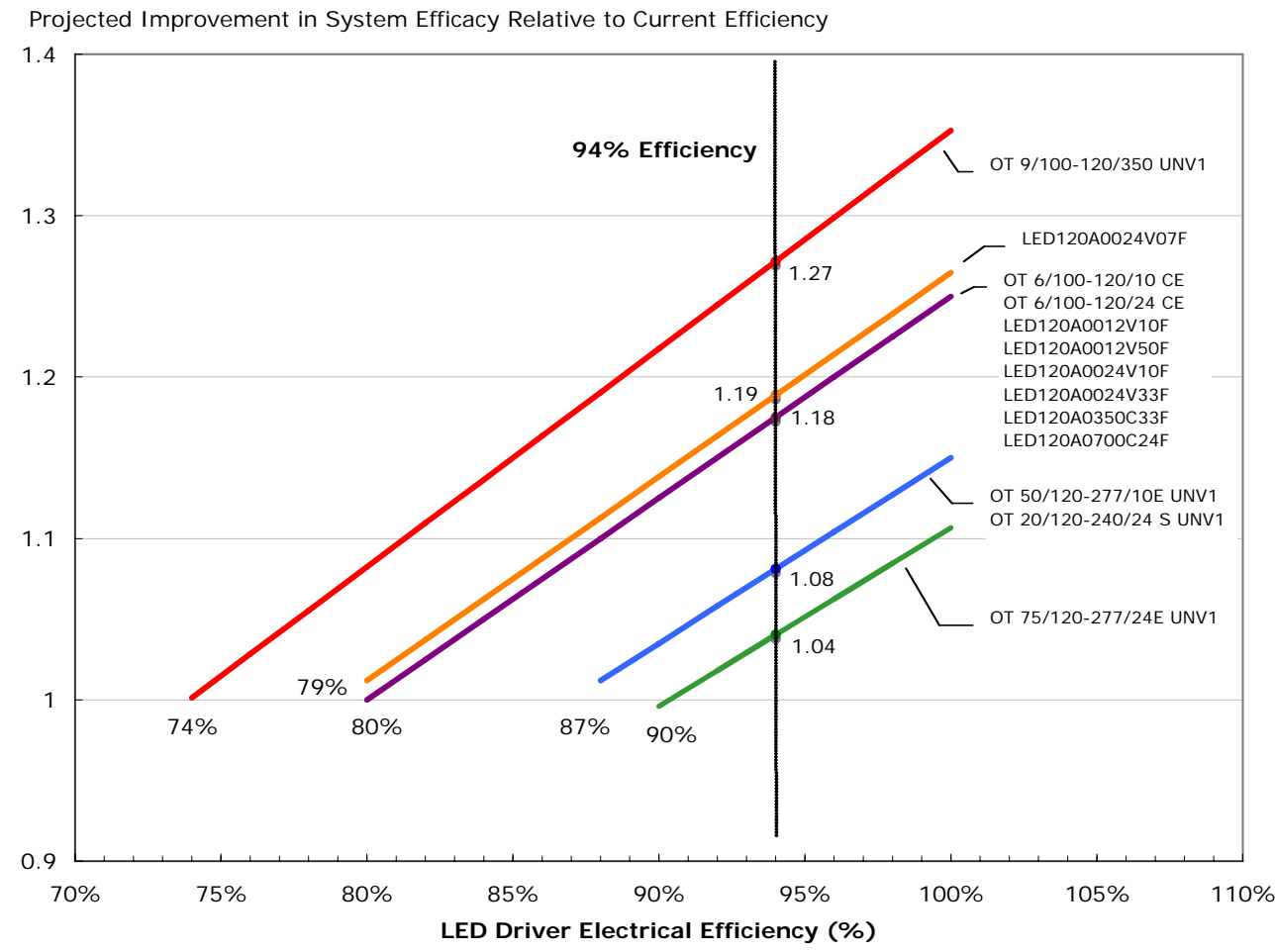

Figure 7. Projected improvement in system luminous efficacy obtained by raising driver electrical efficiency from current indicated levels to $94 \%$ efficiency (maximum practically achievable level)

Reducing driver cost:

Manufacturers can significantly reduce the cost of LED drivers by reducing the electronic parts count on the circuit board. The internal circuit board for a typical $350 \mathrm{~mA}$ constant current driver is shown in Figure 8.

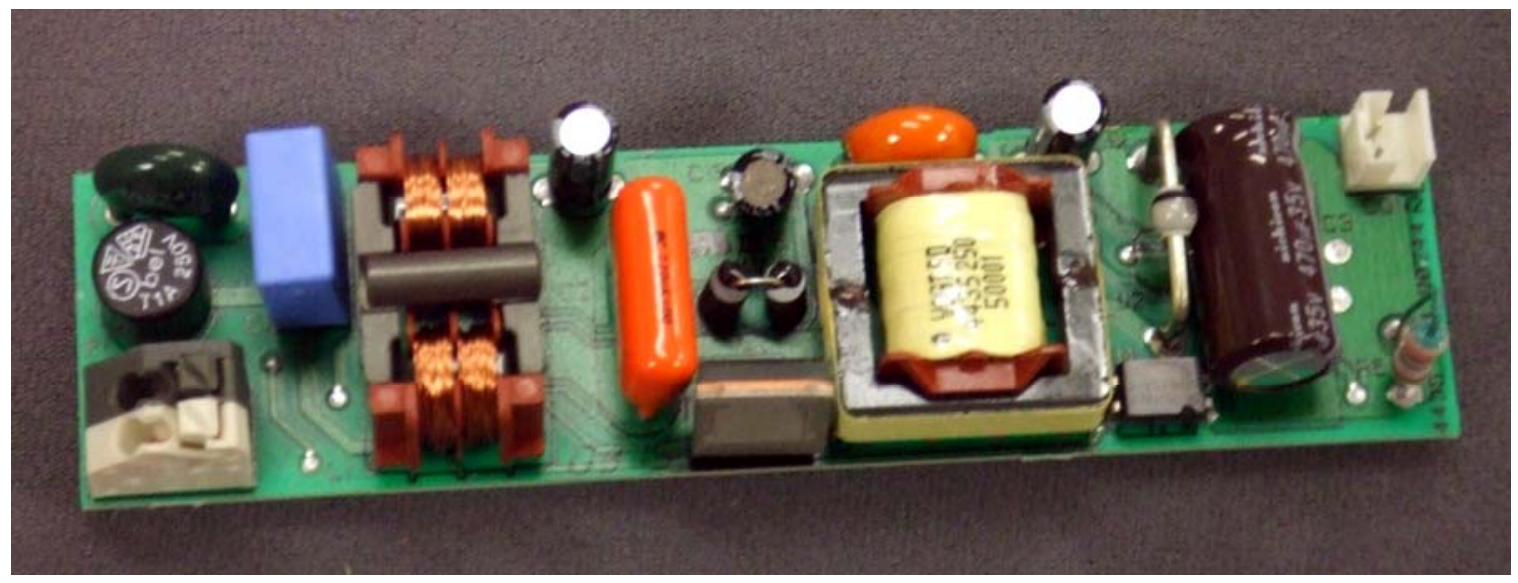

Figure 8. Internal board for Advance LED Driver Model LED120A0350C33F 
For example, examination of a typical LED driver (Advance Model

LED120A0350C33F) shows that it consists of some 60 discrete electronic parts (see Table 2).

Table 2. Parts Inventory for Advance LED120A0350C33F Driver

\begin{tabular}{cl} 
Quantity & \multicolumn{1}{c}{ Part } \\
1 & Power Transistor \\
1 & Small Transistor \\
2 & Transformer \\
3 & Al Electrolytic cap \\
2 & Tantalum cap \\
2 & Film cap \\
8 & Ceramic cap \\
2 & Integrated circuit \\
2 & Bridge rectifier \\
26 & Resistor \\
9 & Diode \\
1 & MOV \\
1 & Fuse \\
60 &
\end{tabular}

Manufacturers can eliminate many of the above components and replace their functionality using integrated circuits (ICs). The simplest possible LED driver need only consist of the following five parts:

- Integrated Circuit

- Inductor

- Capacitor

- Diode

- Resistor

The above is the most extreme parts reduction possible and it is likely that a practical LED driver design will contain at least a dozen discrete components. Nonetheless, a reduced parts driver would represent a significant cost savings to the OEM manufacturer.

\subsection{Interview with Philips Lighting}

To identify Philips Lighting's viewpoint with respect to solid state lighting and lighting controls, researchers conducted a telephone interview with the following individuals on November 10, 2006: 
Ron Steen (Director, Solid State Lighting)

Raj Jayaraman (VP Business Systems)

Jamie Cleveland (Equos Product Manager)

Onno Willemse (Global Program Manager, Solid State Lighting)

Jim Sekinger (Director, Business Development Digital Systems \& Lighting Controls) Key Points:

- Philips strongly supports ZigBee as the wireless intelligent building network of the future.

- ZigBee should significantly reduce the commissioning problem and allow controls to be installed in existing buildings.

- To maximize market space, Philips seeks complete end-to-end controls solutions that are light source neutral (work with fluorescent and incandescent lighting as well as LEDs).

- In Europe there are two "standards" for the DMX cable connectors: either XLR or RJ45. But there is no standardization of the cabling with the Europoean construction industry and issues relating to fire safety and the physical cabling need to be addressed. 


\subsection{Future Requirements}

The previous section discussed the current capabilities of LED drivers and showed how LED driver manufacturers could realize significant cost savings and improve driver efficiency by offloading discrete components onto integrated circuits (IC).

In this section, data is presented on emerging LED controllers and drivers, which boast very high efficiencies for the LED application. Future trends that will influence new LED luminaires are also discussed.

\subsection{Integrated Circuits (ICs) for LED drivers}

Researchers made an exploratory study of the next generation of ICs. For example, Maxim has just announced a new controller for LEDs, the MAX16818 chip, which, when embedded into new LED drivers by original equipment manufacturers (OEMs), promises efficiencies of 94\%. Many chip companies produce these ICs (e.g., Linear Technology, Texas Instruments, Maxim, etc). Unfortunately the raw chips are often referred to as "LED drivers" even though they are not the same as packaged units such as Advance's Xitanium LED drivers or OSI's drivers, which were discussed in the previous section.

Nonetheless, it is instructive to examine the listed efficiencies of these "raw" drivers, because it indicates the magnitude of the efficiency improvements possible in tomorrow's LED drivers. Of the five companies examined, efficiencies varied from 78 to $98 \%$ (see figure below). Of course the efficiency of packaged drivers made from these components will be lower because there are losses associated with the external components and the necessary down-voltage conversion from 120 VAC to 12 or 24 VDC for any practical LED application.

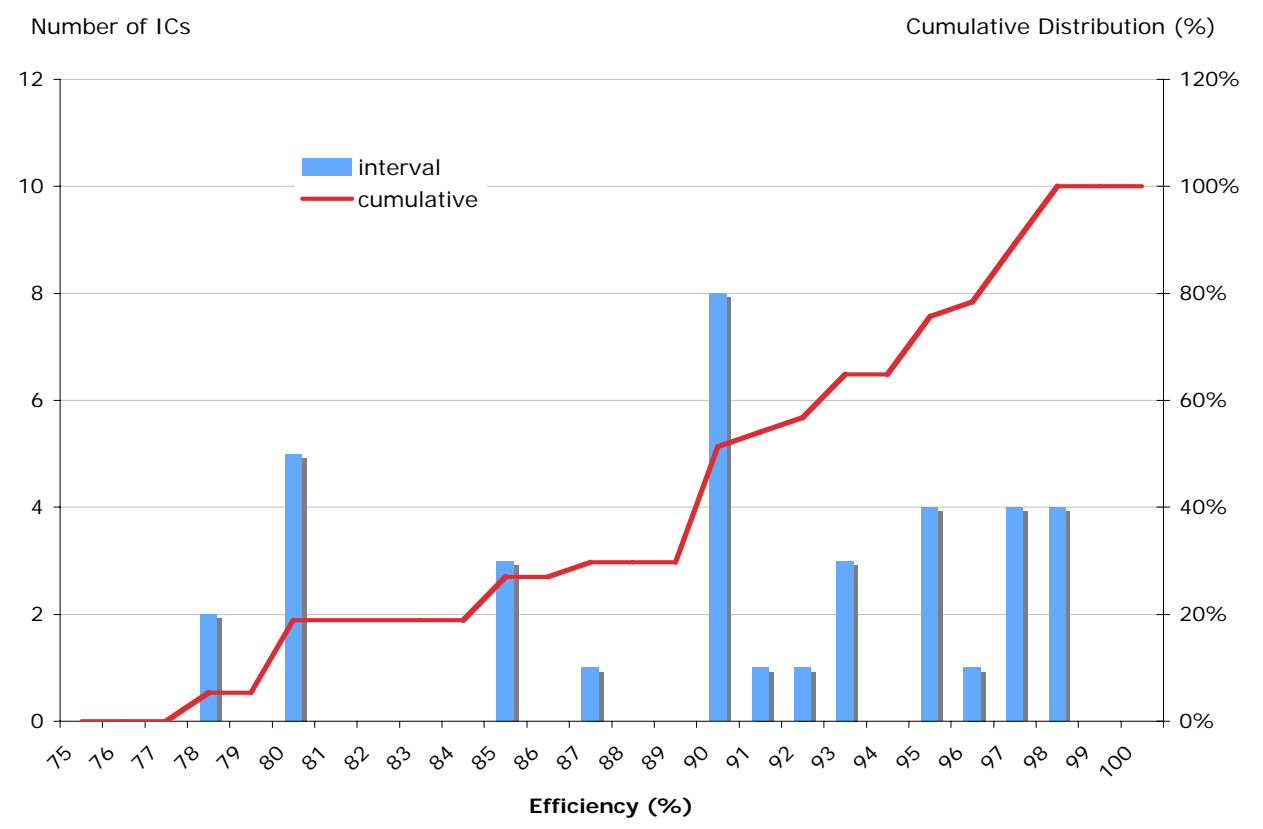

Figure 9. Manufacturer-listed efficiencies of Integrated Circuits (ICs) intended for packaged LED drivers (39 ICs from 5 manufacturers 


\section{2. $\quad$ Future Trends}

LED luminaire manufacturers will increasingly strive to integrate the LED driver and thermal management directly in the LED fixture rather than using discrete LED drivers currently marketed. There are several reasons for this evolution. First, as described earlier, new ICs make it possible to ballast LED clusters very efficiently. Drivers approaching 94\% efficiency are possible with these new ICs and increasing driver efficiency is one method for increasing the luminous efficacy of the LED system. Second, there are functional advantages to integrating the new driver electronics directly into the LED luminaire. Using one heat sink to remove heat both from the LED itself and the driver electronics offers cost advantages. Third, LED luminaires of the future will likely have no need for separable lamp and ballast because the equipment life of all the LED luminaire "components” will all be about the same (50,000 hours). 


\subsection{Market Structure}

\subsection{LED Market}

A 2006 DOE report [1] projects that the LED market for illumination will increase from $\$ 230 \mathrm{M}$ in 2005 to $\$ 800 \mathrm{M}$ in 2010 . Over that same period, LEDs for the signs and displays market will increase from $\$ 500$ million in revenue to over $\$ 3$ billion. Given that signs and displays, especially digital signage and electronic billboards is a new growth market, market players that influence signage and displays will have as great an effect on the emerging LED market as manufacturers of general illumination systems.

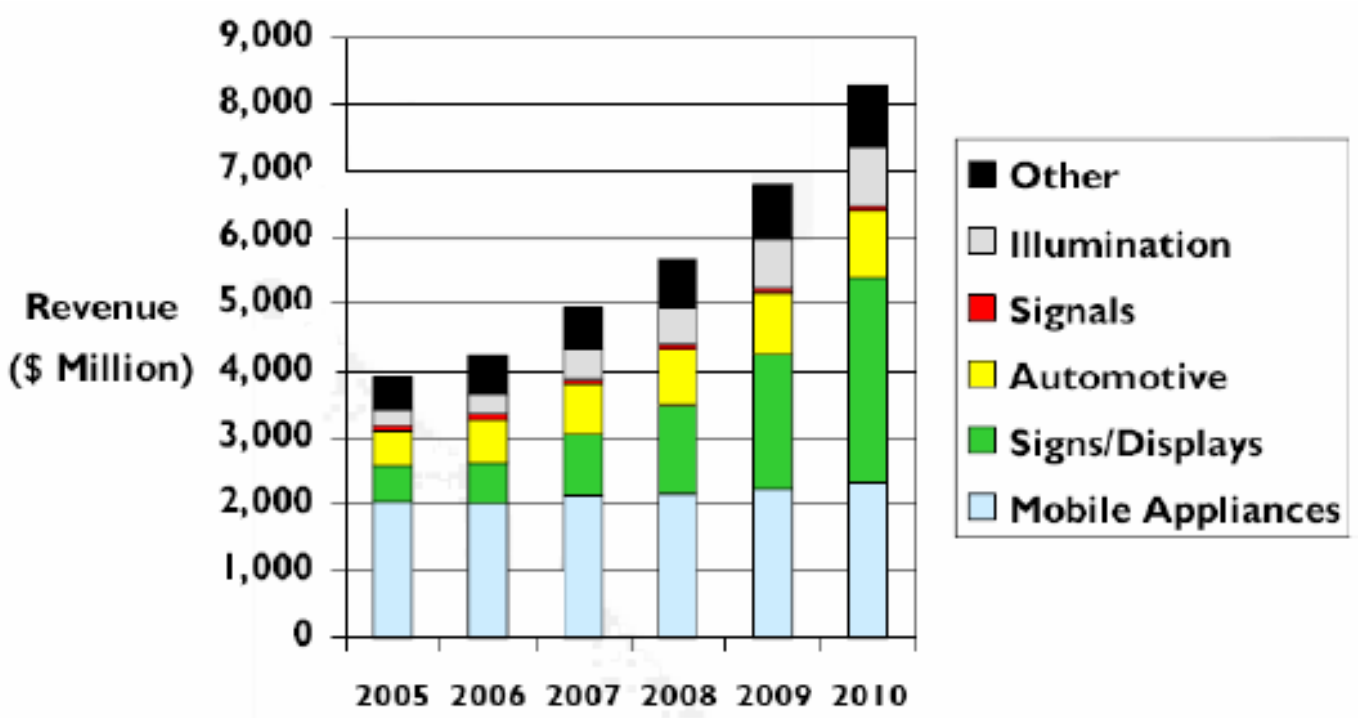

Figure 10. The current distribution of LEDs by major application type (left pie chart) and the expected growth in each of the key market sectors for the next 5 years (right hand plot).

Dorsheimer may well have underestimated the market potential for digital signage and electronic billboards in the US. In January 2007, Cisco systems identified a $\$ 2$ billion market for digital signage by 2010. Not all digital signage uses LEDs, but electronic billboards rely on LEDs and this market is experiencing explosive growth because it is a new lucrative market. Electronic billboards are expected to increase from about 400 units nationally to 4000 units within 3 years and each one successfully commands 10 times the price of non-digital billboards. The primarily RGB LEDs required by the burgeoning billboard market are already cost-effective at today's prices for these applications.

\subsection{LED Market Players}

This section presents the market structure of the controls suppliers for LED illumination systems. We consider primarily the market for high-brightness white LEDs and associated controls used for the purpose of efficiently managing lighting energy usage. The LED market for commercial lighting consists of the following entities: 
Table 3. LED Market Entities and Main Market Functions

\begin{tabular}{ll}
\hline Entity & Market function \\
\hline High-Brightness LEDs & White LED packages \\
Controls & $\begin{array}{l}\text { Communications, dimming } \\
\text { LED drivers (including chip sets) and ballasts } \\
\text { (packaged drivers) }\end{array}$ \\
Integrators & Integration and packaging \\
Modules & Light engines and assemblies \\
Optics & Lenses and light pipes \\
Passives & Heat sinks \\
Thermal & Metal PCBs, fans \\
\hline
\end{tabular}

In terms of the controls issues facing LED lighting, the controls manufacturers and driver manufacturers are the most significant players. That is, their product lines and strategic approach will most affect the role of conventional "controls" in the LED lighting in the future. While there are also controls issues related to thermal management and color consistency of the LED modules, these intra-fixture controls issues are the purview of the integrators and luminaire manufacturers. While manufacturers will apply proprietary control techniques to remove heat from LED junctions, there is little need for open communications and controls within fixture .

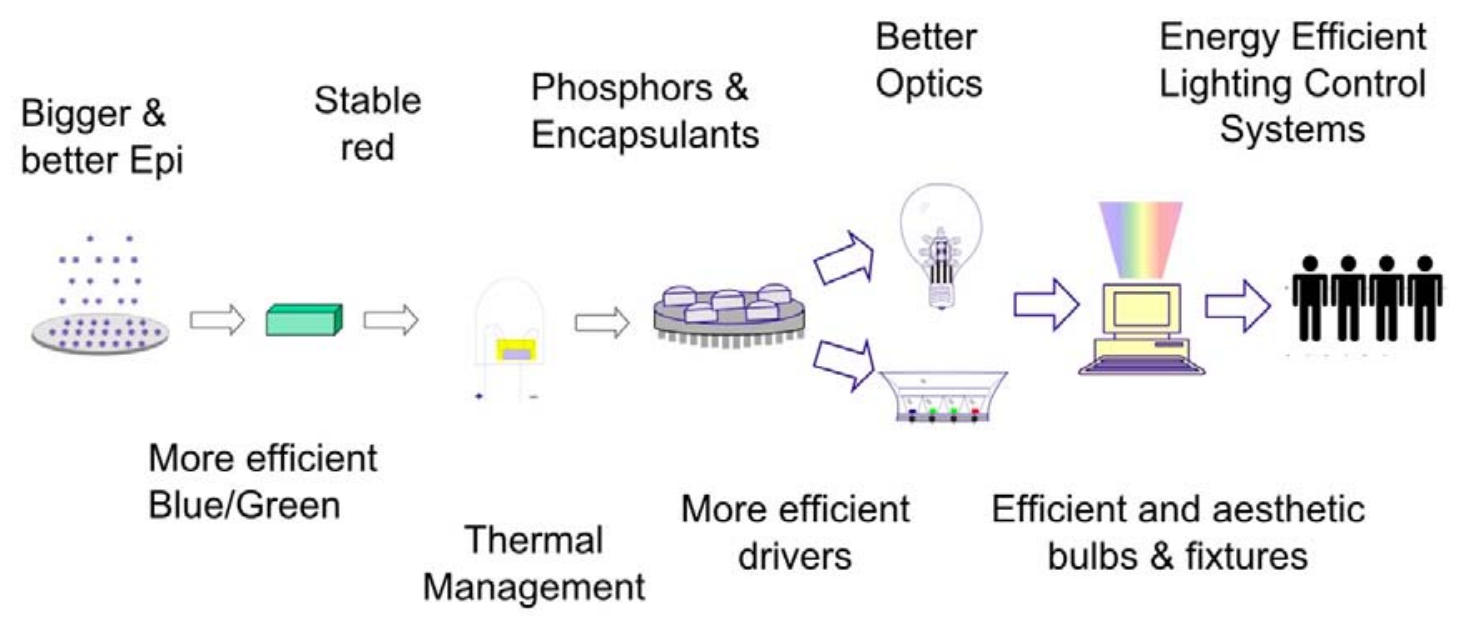

Figure 11. LED Market Chain showing manufacturing steps from wafer to final lighting system (From Thurk 2006) 
Most of the important challenges for lighting controls relative to LEDs and energy efficiency concern the intelligent operation of groups of LED luminaires (inter-fixture control) analogous to communications and controls for advanced fluorescent lighting systems.

One trend that we identified earlier in the report is the development of hybrid LED systems which combine a "conventional” light source, such as fluorescent or even incandescent lamp, that is motion-sensor triggered and a low-energy "standby" level provided by LEDs. These hybrid LED systems take advantage of a growing awareness that lighting systems that can only switch on and off are not adequate to today's modern life and need for security. In many commercial building spaces, including stairwells, service corridors, general corridors and building exteriors, a low-energy "standby" level of light is desirable all the time. ${ }^{3}$

All of the basic components necessary for fixture OEMs to produce cost-effective hybrid LED systems are available today. There are numerous manufacturers of lighting control equipment today and, increasingly, manufacturers are providing entire end-to-end solutions, not just individual control components. Manufacturers of hybrid LED systems have many vendors to choose, many with rich product lines covering all control components.

\footnotetext{
${ }^{3}$ Fire marshalls in many municipalities do not want fire workers to enter unlit stairwells. Bi-level stairwell fixtures combine standard fluorescent lighting with a low-energy "standby” level that provides a low level of illumination all the time. The fixture goes to full output when the fixture detects motion in its vicinity.
} 


\subsection{Findings and Conclusions}

This study has brought to light new applications for LEDs that combine the LED source for one light level with another, more powerful, efficient light source for occupied periods. It is the lighting controls which allow these two light sources to work together through an appropriate control strategy, typically motion-sensing. There are no technical barriers preventing the use of low-high CRI LEDs for standby lighting in many interior and exterior applications today.

The study finds that the total luminous efficacy of LED systems could be raised by increasing the electrical efficiency of LED drivers to the maximum practically achievable level (94\%). This would increase system luminous efficacy by $20-25 \%$. The expected market volumes for many types of LEDs should justify the evolution of new LED drivers that use highly efficient ICs and reduce parts count by means of ASICs. Reduce their electronics parts count by offloading discrete components onto integrated circuits (IC) should reduce the cost of LED driver electronics.

The study predicts that LED luminaire manufacturers will increasingly strive to integrate the LED driver and thermal management directly in the LED fixture. LED luminaires of the future will likely have no need for separable lamp and ballast because the equipment life of all the LED luminaire "components" will all be about the same (50,000 hours).

The study finds that the controls and communications techniques used for communicating with conventional light sources, such as dimmable fluorescent lighting, are appropriate for LED illumination as well at least as far as energy management in concerned. DALI has been used to control LED systems in some new applications and the emerging ZigBee protocol could be used for LEDs as well. Indeed, the major lighting companies are moving in this direction.

The most significant finding is that there is a significant opportunity to use LEDs today for standby lighting purposes. Conventional lighting systems can be made more efficient still by using LEDs to provide a low-energy standby state when lower light levels are acceptable. 


\section{References}

[1] DOE, 2006. Energy Savings Potential of Solid State Lighting in General Illumination Applications. Final Report, prepared by Navigant Consulting, Inc. for the U.S. Department of Energy, Office of Energy Efficiency and Renewable Energy, Building Technologies Program, Washington DC, December 2006.

[2] DOE, 2002. U.S. Lighting Market Characterization, Volume I: National Lighting Inventory and Energy Consumption Estimate. Final Report, prepared by Navigant Consulting, Inc. for the U.S. Department of Energy, Office of Energy Efficiency and Renewable Energy, Building Technologies Program, Washington DC, September 2002.

[3] Thurk, Paul, “Raising Money for Solid State Lighting, General Illumination”, presentation made to SSL Roundtable, Feb, 2007.

[4] Dorsheimer, Jed, “A Bright Idea: Opportunities and Challenges in Solid State Lighting”, presentation made at Controlling Light 2006 conference, San Diego December 2006.

[5] DOE, 2005. 2005 Buildings Energy Databook, Office of Energy Efficiency and Renewable Energy, U.S. Department of Energy, Washington DC, 2005.

[6] Washington Post, “Billboards vs. a greener Nation”, May 2, 2007.

[7] Next Generation Lighting Industry Alliance, "NGL Industry Alliance Handout Sheet", Sept. 112003

[8] Rubinstein, F, The Usefulness of Bi-Level Switching, Lawrence Berkeley National Laboratory. Technical Note, LBNL-44281, Revised August 1999

[9] Gu, Y, N. Narendran, T Dong and H Wu, "Spectral and Luminous Efficacy Change of High-Power LEDs under Different Dimming Methods”, Sixth International Conference on Solid State lighting, Proceedings of SPIE 6337, 63370J 


\section{Appendix A - Statement of Work}

\section{A.1 Project Objectives}

The objective of the project is to characterize and identify trends towards control, operation, and maintenance of Solid State Lighting systems, both today and as envisioned for tomorrow's lighting systems. The approach shall include a market reconnaissance study to identify the technical and market issues associated with the control, operation and maintenance of solid state lighting systems. The study scope is primarily limited to the control of LED sources but may include analysis of OLEDs as appropriate.

\section{Task 1 - Current Capabilities}

The Recipient shall investigate current industry capabilities with respect to the control of LED sources. As a result, the Recipient shall identify capabilities, strengths, and weaknesses of current products and equipment with respect to dimming (intensity adjustment), color consistency (intra- and inter-fixture) and color temperature. The Recipient shall detail the importance of controls for maintaining source color temperature. The Recipient shall identify requirements for SSL controls equipment including a discussion of different modes of operation, voltage and current limitations for transformers and IC-based power conversion control gear. The Recipient shall compare and contrast market available control gear with respect to size, cost, and performance.

\section{Task 2 - Future Requirements}

The Recipient shall identify appropriate market drivers that will influence future LED control requirements. This shall include a discussion of requirements for future controls equipment and transformers, including different modes of operation (DC, pulse width modulation, etc), voltage and current limitations. The Recipient shall investigate how controlled SSL systems will integrate with existing lighting systems in standing building stock and new construction. The Recipient shall explore future expected trends in modern building lighting. The Recipient shall identify technical challenges associated with controlling LEDs from wireless or power-line carrier communications systems.

\section{Task 3 - Market Structure}

The Recipient shall describe the structure of the current market, identify the current controls suppliers and the relationships between them (value chain). The Recipient shall also describe the value chain, identifying the roles of all the major players. The Recipient shall identify the size of the current market, project the increase in market size if market transformation is achieved, and detail the changing influences on the roles of the major market players as LEDs mature. 